\section{Mass Spectrometry in the Biological Sciences}

A. L. Burlingame and S. A. Carr, Editors

Humana Press, Totowa, New Jersey

1996, 570 p., \$145

Book review by Daniel R. Knapp

Department of Pharmacology

Medical University of South Carolina .

Charleston, SC 29425

This book derives from the Third International Symposium on Mass Spectrometry in the Health and Life Sciences held in San Francisco, CA, September 13-18, 1994. Presented as a part of the education and dissemination function of the National Institutes of Health supported University of California at San Francisco (UCSF) Biomedical, Bio-organic Mass Spectrometry Resource, this meeting and the resulting printed volume provide a summary of the status of the application of mass spectrometry in a variety of areas of biology. The book is largely, although not exclusively, devoted to biopolymer analysis which includes proteins and peptides, carbohydrates, and nucleic acids. Unlike many symposium volumes, the 25 papers, which average 21 pages in length and more than 30 references each, are sufficiently detailed and documented to be of significant reference value. Author and subject indexes also are included. Of further interest are the printed questions and answers from the discussion that followed each presentation at the meeting.

An uncommon feature of the book is a series of 10 appendixes that list useful reference data for mass spectrometry of peptides and carbohydrates. An eleventh appendix is a referenced discussion by the editors titled "The Meaning and Usage of the Terms Monoisotopic Mass, Average Mass, Mass Resolution, and Mass Accuracy for Measurements of Biomolecules." This brief primer, which also addresses the question "how much resolution is required?," should be of help to sort out these sometimes confusing terms, especially for newcomers in biopolymer mass spectrometry.

An introductory statement by J. Vaitukaitis and $M$. Mylander of the National Institutes of Health National Center for Research Resources (NCRR) speaks to the importance of mass spectrometry in contemporary biomedical research and to the commitment of the NCRR to make this and other critical technologies available to researchers, but also points out the necessity of strategic planning and priority setting to maximize yields from the limited funds available. Mass spectrometrists are challenged to devise ways to make modern mass spectrometry approaches available to more researchers in the future. Fortunately, this is already happening as evidenced, for example, by the availability via the Internet of the MOWSE (molecular weight search) database, as described by D. J. C. Pappin et al. in the book and of similar capabilities via the UCSF Mass Spectrometry Resource Web page.

Papers by R. Cotter, et al. and by R. D. Smith et al., respectively, discuss new developments in time-offlight and Fourier transform ion cyclotron resonance mass spectrometry as applied to biological research. Also discussed are the observations of covalent, ionic and noncovalent interactions by electrospray ( $R$. T. Aplin and C. V. Robinson) and the use of deuterium exchange to study protein structure (R. Anderegg). Four papers are devoted to the approaches of the authors' laboratories to identify proteins on polyacrylamide electrophoresis gels. Several of the authors describe particular applications of mass spectrometry to protein and peptide studies in the laboratories including, for example, neuropeptide profiling (C. R. Jimenez et al.) major histocompatibility complex (MHC)-bound antigenic peptides (P. H. Gulden et al.), and a potential recombinant vaccine (M. E. Hemling et al.). Other papers describe a variety of different applications from the authors' laboratories. Four papers discuss carbonhydrate analysis (A. M. Lawson et al., lipooligosaccharides (W. Melaugh et al.). Only one of the papers deals primarily with nucleic acid analysis (P. F. Crain et al.), although the final paper is a discussion of the future of DNA analysis by C. R. Cantor et al. Papers devoted to applications other than biopolymers include those that relate to drug biotransformation studies (D. M. Dulick et al.), isoeicosanoids ( $R$. Murphy et al.), and accelerator isotope analysis (K. W. Turteltaub and J. S. Vogel).

Although much of the content is now superseded by more recent journal articles, the book nonetheless is of reference value. Unfortunately, the high cost will limit its availability. Libraries are increasingly reluctant to expend their limited acquisitions funds on symposium volumes. Those persons who wish to convince their local librarians to acquire this volume might cite the appendixes in particular as a feature that sets this book apart from the usual symposium volumes. 